\title{
Exactly solvable three-body Calogero-type model with translucent two-body barriers
}

\author{
Miloslav Znojil and Miloš Tater \\ oddělení teoretické fyziky, \\ Ústav jaderné fyziky AV ČR, 25068 Řež, Czech Republic円
}

\begin{abstract}
A new exactly solvable alternative to the Calogero three-particle model is proposed. Sharing its confining long-range part, it contains the mere zero-range two-particle barriers. Their penetrability gives rise to a tunneling, tunable via their three independent strengths. Their variability can control the removal of the degeneracy of the energy levels in an innovative, non-perturbative manner.
\end{abstract}

PACS 21.45.+v 03.65.Ge 02.60.Lj

\footnotetext{
${ }^{1}$ e-mail: znojil@ujf.cas.cz and tater@ujf.cas.cz
} 


\section{Introduction}

In nuclear, atomic, molecular and statistical physics, the enormous popularity of the Calogero's solvable models [1] reflects a fairly realistic form of their separate twobody interactions. They combine a long-range quadratic attraction with a strong short-range repulsion. In its full generality the model binds an arbitary number $N$ of particles moving along a straight line and its exact solvability is a consequence of certain deep symmetries of its partial differential Schrödinger equation [2].

For the sake of brevity, let us pay attention just to the Calogero's first nontrivial three-body Hamiltonian

$$
\begin{aligned}
H^{(C a l)}=-\frac{\hbar}{2 m}\left[\frac{\partial^{2}}{\partial x_{1}^{2}}+\frac{\partial^{2}}{\partial x_{2}^{2}}+\frac{\partial^{2}}{\partial x_{3}^{2}}\right]+ \\
+\frac{1}{8} \omega^{2}\left[\left(x_{1}-x_{2}\right)^{2}+\left(x_{2}-x_{3}\right)^{2}+\left(x_{3}-x_{1}\right)^{2}\right]+ \\
+\left[\frac{g_{1}}{\left(x_{2}-x_{3}\right)^{2}}+\frac{g_{2}}{\left(x_{3}-x_{1}\right)^{2}}+\frac{g_{3}}{\left(x_{1}-x_{2}\right)^{2}}\right] .
\end{aligned}
$$

We may immediately see that from a purist's point of view, its two-body barriers are not penetrable [3] and, in this sense, they do not admit any mutual exchange of particles. For this reason we proposed recently a solvable modification of this model where a tunneling has been rendered possible at an expense of a loss of the Hermiticity of the Hamiltonian $H^{(\mathrm{Cal})}$ [4]. We employed a complexification based on the shift of the coordinates $x^{(r e a l)} \rightarrow x^{(\text {complex })}(t)=t-i \varepsilon\left(t^{2}\right)$. This replaced the Hermiticity of the Hamiltonian by its mere commutativity with the product of parity $\mathcal{P}$ and complex conjugation $\mathcal{T}$ [5]. We have shown that the spectrum remained real in a way attributed to the $\mathcal{P} \mathcal{T}$ symmetry in the related literature [6].

In the latter innovative few-body implementation of the idea of the $\mathcal{P} \mathcal{T}$ symmetrization, the Calogero's strongly singular real barriers $1 / x^{2}$ with $x=x_{j}-x_{k}$ were all replaced by the complex and $\varepsilon$-dependent expressions

$$
\frac{1}{(x-i \varepsilon)^{2}}=\frac{1}{x^{2}+\varepsilon^{2}}+\frac{2 i \varepsilon x}{\left(x^{2}+\varepsilon^{2}\right)^{2}}+\mathcal{O}\left(\varepsilon^{2}\right) .
$$

In the present short note we intend to describe and analyze an alternative scheme 
which would admit a tunneling. In essence, we shall start from the same leading-order formula (2) but succeed in returning to a Hermitian Hamiltonian.

\section{The new model}

\subsection{Inspiration}

Our new proposal has been inspired by formula (2) and by its Hermitization

$$
\frac{1}{x^{2}} \rightarrow \frac{1}{x^{2}+\varepsilon^{2}}=\frac{1}{2 i \varepsilon}\left[\frac{1}{x-i \varepsilon}-\frac{1}{x+i \varepsilon}\right]=\frac{\pi}{\varepsilon} \tilde{\delta}_{\varepsilon}(x), \quad \varepsilon>0 .
$$

The limit $\varepsilon \rightarrow 0$ would then just reproduce the Calogero's model, one of the specific and most inspiring features of which lies in its separability in the three-body case. This is based on the re-parametrization of the coordinates $x_{1}-x_{2}=\sqrt{2} \rho \sin \phi$ with $\rho \in(0, \infty)$ and $\phi \in(0,2 \pi)$ etc (cf. Figure 1$)$. In the units $2 m=\hbar=1$ and for the equal and non-negative coupling constants $g_{1}=g_{2}=g_{3}=g \geq 0$ in eq. (1), such a change of variables reduces the Calogero's partial differential Schrödinger eqution to the mere ordinary Sturm - Liouvillean problem

$$
\left(-\frac{d^{2}}{d \phi^{2}}+\frac{9 g}{2 \sin ^{2} 3 \phi}\right) \psi(\phi)=\kappa^{2} \psi(\phi), \quad \psi(0)=\psi(2 \pi)=0 .
$$

It solution generates the well known (viz., Laguerre times Jacobi) polynomial wave functions as well as the related equidistant spectrum with a gap, $E_{n} \sim n+$ const, $n=0,2,3,4, \ldots$

\subsection{Main idea}

Our attention is attracted by the tilded expression $\tilde{\delta}_{\varepsilon}(x)$ in eq. (3). We shall try to re-read it as an approximate $\varepsilon \approx 0$ form of the well known Dirac delta function $\tilde{\delta}_{0}(x)=\delta(x)$. Thus, picking up, say, the first part of the two-body barrier in eq. (11) in its regularized form

$$
\frac{g_{1}}{\left(x_{2}-x_{3}\right)^{2}+\varepsilon^{2}}=f_{1} \tilde{\delta}_{\varepsilon}\left(x_{2}-x_{3}\right), \quad f_{1}=\frac{\pi g_{1}}{\varepsilon}, \quad \varepsilon>0
$$


we may insert the appropriate definition $x_{2}-x_{3}=\sqrt{2} \rho \sin (\phi-4 \pi / 3)$ and get, at the very small $\varepsilon \rightarrow 0$,

$$
f_{1} \tilde{\delta}_{\varepsilon}\left(x_{2}-x_{3}\right) \approx \frac{G_{1}}{\rho^{2}}\left[\tilde{\delta}_{\varepsilon}\left(\phi-\frac{\pi}{3}\right)+\tilde{\delta}_{\varepsilon}\left(\phi-\frac{4 \pi}{3}\right)\right], \quad G_{1}=\frac{f_{1} \rho}{\sqrt{2}} .
$$

In place of (4) it gives

$$
\begin{aligned}
&-\frac{d^{2}}{d \phi^{2}} \psi(\phi)+ G_{1}\left[\tilde{\delta}_{\varepsilon}\left(\phi-\frac{\pi}{3}\right)+\tilde{\delta}_{\varepsilon}\left(\phi-\frac{4 \pi}{3}\right)\right] \psi(\phi)+ \\
&+G_{2}\left[\tilde{\delta}_{\varepsilon}\left(\phi-\frac{2 \pi}{3}\right)+\tilde{\delta}_{\varepsilon}\left(\phi-\frac{5 \pi}{3}\right)\right] \psi(\phi)+ \\
&+G_{3}\left[\tilde{\delta}_{\varepsilon}(\phi-\pi)+\tilde{\delta}_{\varepsilon}(\phi)\right] \psi(\phi)=\kappa^{2} \psi(\phi),
\end{aligned}
$$

i.e., an approximative innovation of the Calogero's angular Schrödinger equation.

\subsection{Interpretation}

Due to the purely intuitive form of the above "derivation" of eq. (5), one has to be very careful in all questions related to its possible physical as well as mathematical interpretation. At the same time, the use of the delta-function-shaped potentials is quite common in practice [7] as it makes many systems exactly solvable in the limit $\varepsilon \rightarrow 0$.

Let us start our further analysis of eq. (5) by picking up some three constants $G_{k}>0$. Then we re-define $f_{k}=f_{k}(\rho)=\sqrt{2} G_{k} / \rho$ and $g_{k}=\varepsilon f_{k}(\rho) / \pi$. In the generic case with $x_{i}-x_{j} \neq 0$, the limiting transition to $\varepsilon=0$ makes all the three centrifugallike forces vanish, $g_{k} \rightarrow 0$. Such an observation is compatible with the philosophy of using just the contact barriers in our angular Schrödinger equation.

In the second step we have to re-analyze the role of the overall singularity in the origin $\rho=0$. In principle, we might admit and consider many different singularities there [9]. Here we shall simplify our life by the most straightforward postulate that the point $\rho=0$ is just "regular" (i.e., "ignored" by our Hamiltonian), and that all our radial wave functions are simply vanishing in the origin. Under such an assumption, the final $\rho \neq 0$ form of our new Hamiltonian $H^{(n e w)}$ can be assigned a 
formal representation

$$
\begin{aligned}
H^{(\text {new })}=- & \frac{\hbar}{2 m}\left[\frac{\partial^{2}}{\partial x_{1}^{2}}+\frac{\partial^{2}}{\partial x_{2}^{2}}+\frac{\partial^{2}}{\partial x_{3}^{2}}\right]+ \\
+\frac{1}{8} \omega^{2} & {\left[\left(x_{1}-x_{2}\right)^{2}+\left(x_{2}-x_{3}\right)^{2}+\left(x_{3}-x_{1}\right)^{2}\right]+} \\
+ & \Omega_{1} \delta\left(x_{2}-x_{3}\right)+\Omega_{2} \delta\left(x_{3}-x_{1}\right)+\Omega_{3} \delta\left(x_{1}-x_{2}\right)
\end{aligned}
$$

where the strength of the two-body contact terms has the three-body character and weakens with the distance of the detached spectator particle,

$$
\Omega_{1}=\frac{\sqrt{3} G_{1}}{\left|x_{1}-x_{2}\right|}, \quad \Omega_{2}=\frac{\sqrt{3} G_{2}}{\left|x_{2}-x_{3}\right|}, \quad \Omega_{3}=\frac{\sqrt{3} G_{3}}{\left|x_{3}-x_{1}\right|} .
$$

We may summarize that our Hamiltonian $H^{(n e w)}$ represents a new three-particle model which is exactly solvable.

\section{Solutions}

Our new angular Schrödinger equation

$$
\begin{aligned}
-\frac{d^{2}}{d \phi^{2}} \psi_{k}(\phi)+ & G_{1}\left[\delta\left(\phi-\frac{\pi}{3}\right)+\delta\left(\phi-\frac{4 \pi}{3}\right)\right] \psi_{k}(\phi)+ \\
+G_{2}\left[\delta\left(\phi-\frac{2 \pi}{3}\right)+\delta\left(\phi-\frac{5 \pi}{3}\right)\right] \psi_{k}(\phi)+ & \\
& +G_{3}[\delta(\phi-\pi)+\delta(\phi)] \psi(\phi)=\kappa_{k}^{2} \psi_{k}(\phi)
\end{aligned}
$$

describes the $\phi$-dependent part of the three-body wave function and determines its energies via the Calogero-type formula,

$$
E=E_{m, k}=\sqrt{\frac{3}{2}} \omega\left(2 m+1+\kappa_{k}\right), \quad m, k=0,1, \ldots .
$$

Within the open subintervals of $\phi \in \mathcal{D}_{j}=([j-1] \pi / 3, j \pi / 3)$ we have a free motion

$$
\psi(\phi)=\psi_{j}(\phi)=A_{j} \sin \{\kappa[\phi-(j-1) \pi / 3]\}+B_{j} \cos \{\kappa[\phi-(j-1) \pi / 3]\} .
$$

On the boundaries we have to match the separate local wave functions using the standard rules

$$
\psi_{j}\left(\frac{j \pi}{3}\right)=B_{j+1}, \quad \partial_{\phi} \psi_{j}\left(\frac{j \pi}{3}\right)=\kappa\left[A_{j+1}+\beta_{j}(\kappa) B_{j+1}\right], \quad j=1,2, \ldots, 6
$$


with $\beta_{j}(\kappa)=\beta_{j+3}(\kappa)=G_{j} / \kappa$ and $j=1,2$ or 3 . The first five applications of these rules define all the wave functions $\psi_{j}(\phi)$ in terms of the initial one, say, $\psi_{1}(\phi)$. The sixth step becomes a selfconsistent matching which guarantees that the global wave function remains single-valued. In terms of the two auxiliary matrices

$$
R=R(\kappa)=\left(\begin{array}{cc}
\cos (\kappa \pi / 3) & \sin (\kappa \pi / 3) \\
-\sin (\kappa \pi / 3) & \cos (\kappa \pi / 3)
\end{array}\right), \quad L=\left(\begin{array}{cc}
0 & 0 \\
1 & 0
\end{array}\right)
$$

our matching conditions factorize in the two independent two-dimensional forms

$$
(\mathcal{U} \pm I)\left(\begin{array}{c}
B_{1} \\
A_{1}
\end{array}\right)=0
$$

where $\mathcal{U}=\mathcal{U}(\kappa)=\left(I+\beta_{3} L\right) R\left(I+\beta_{2} L\right) R\left(I+\beta_{1} L\right) R$. The pertaining two alternative two-dimensional secular equations

$$
\operatorname{det}\left[\mathcal{U}\left(\kappa^{( \pm)}\right) \pm I\right]=0
$$

define all the roots $\kappa_{k}^{(\sigma)} \geq 0$ with the sign ambiguity $\sigma= \pm 1$ and the angular quantum number $k=0,1, \ldots$ in implicit manner.

\subsection{Toy model with the single barrier}

Let us fix $G_{1}=G_{2}=0$ and vary the strength $G=G_{3}$. With $\beta=G / \kappa^{(\sigma)}$ in the matrix

$$
\mathcal{U}^{(t o y)}(\kappa)=(I+\beta L) \cdot\left(\begin{array}{cc}
\cos \kappa \pi & \sin \kappa \pi \\
-\sin \kappa \pi & \cos \kappa \pi
\end{array}\right)
$$

our determinantal secular equation reads

$$
\cos \kappa \pi+\sigma+\frac{G \sin \kappa \pi}{2 \kappa}=0
$$

At $\sigma=-1$ and any integer shift of $\kappa^{(-)}=2 k+\delta$ with $k=0,1, \ldots$, this secular equation gives us two roots $\delta(a), \delta(b) \in[0,1)$. The smaller one is trivial, $\delta(a)=0$. The second one is uniquely specified by the implicit formula

$$
\tan \frac{\pi \delta(b)}{2}=\frac{G}{4 k+2 \delta(b)}
$$


Also for $\sigma=+1$ and $\kappa^{(+)}=2 k+1+\delta$ we get $\delta(a)=0$ and the similar relation

$$
\tan \frac{\pi \delta(b)}{2}=\frac{G}{4 k+2+2 \delta(b)}
$$

In the limit $G \rightarrow 0$ of the vanishing barrier we get the standard square-well solutions with the correct degeneracy $\delta(b) \rightarrow \delta(a)$ at every $k$.

The sign of the coupling depends on our choice, $G \in(-\infty, \infty)$. This provides an interesting counterpart to the Calogero model where the barrier cannot be too attractive [10]. In the limit $G \rightarrow \infty$ of the very strong repulsion, one returns to the Calogero-like case characterized by the impenetrability of the barriers.

\subsection{Three equal barriers}

When we choose $G_{1}=G_{2}=G_{3}=G$ and abbreviate $(I+\beta L) R=\mathcal{R}(\kappa)$ with $\beta=G / \kappa$, our secular equation (9) may be factorized into the two separate conditions at both the quasi-parities $\sigma= \pm 1$,

$$
\operatorname{det}[\mathcal{R}(\kappa)+\sigma I]=0, \quad \operatorname{det}\left[\mathcal{R}^{2}(\tilde{\kappa})-\sigma \mathcal{R}(\tilde{\kappa})+I\right]=0
$$

The first one parallels our preceding toy model and its solution is immediate,

$$
\kappa(a)=3 N, \quad \kappa(b)=3 N+3 \delta(b), \quad N=0,1, \ldots
$$

The implicit definition of the pertaining shifts $\lambda(b) \in(0,1)$ has almost the same form as above,

$$
\tan \frac{\pi \delta(b)}{2}=\frac{G}{6 N+6 \delta(b)} .
$$

In the tilded case, the identity $\operatorname{det}\left(\mathcal{R}^{2}-\sigma \mathcal{R}+I\right)=(\sigma-\operatorname{tr} \mathcal{R})^{2}$ holds as long as $\operatorname{det} \mathcal{R}=1$. This reduces the second secular condition to the trigonometric equation

$$
2 \cos \frac{\tilde{\kappa} \pi}{3}+\frac{G}{\tilde{\kappa}} \sin \frac{\tilde{\kappa} \pi}{3}=\sigma
$$

Its solutions must be sought numerically, giving $\tilde{\kappa}=1.367840720,2.199769250, \ldots$ at $G=1$ (cf. Figure 2). 


\section{Summary}

One of the most striking features of the model of Calogero is the fully impenetrable character of its two-body repulsive barriers. They divide the phase space in the six independent subdomains (cf. Figure 1). In a way, this absolute impenetrability of the barriers is in its effect responsible for the exact solvability of the Calogero's model.

This role of the Calogero's $g x^{-2}$ barriers is partially weakened when they become attractive, i.e., $0>g>-1 / 4$. An extension of the Calogero model to this transition region has been discovered and described by Gangopadhyaya and Sukhatme [8]. In their construction the contact terms also appeared as a formal means of preservation of the solvability. As a consequence, the spectrum only consisted of the two shifted sets of equally spaced energy levels. Such a type of the modified Calogero's spectrum re-appeared also in our recent non-Hermitian construction [4].

In the present letter we were able to get rid of the Calogero's power-law $\mathcal{O}\left(x^{-2}\right)$ barriers completely. Our alternative way of introduction of the contact terms enabled us to treat their couplings as independent parameters. Our key point is that these barriers are "thin", i.e., partially penetrable. This represents their main phenomenological appeal. At all their finite (and, in fact, both repulsive as well as attractive) couplings $G_{j}$, their free variability might prove appealing in many phenomenological considerations.

In contrast to the Calogero model characterized by the absolute absence of tunneling, all our particles are permitted to jump over one another. In certain applications to few body systems of quark type, this could improve our intuitive insight and build some analogies with the motion in more dimensions. After all, the use of the harmonic two-body forces with an additional, contact "local spike" might also extend the advantages of the exact solvability quite easily beyond the traditional domains in the theoretical nuclear physics. 


\section{References}

[1] F. Calogero, J. Math. Phys. 10 (1969) 2191 and 2197 and 12 (1971) 419.

[2] W. Rühl and A. Turbiner, Mod. Phys. Lett. A 10 (1995) 2213;

A. Turbiner, Mod. Phys. Lett. A 13 (1998) 1473;

A. Khare and C. Quesne, Phys. Lett. A 250 (1998) 33;

F. Cannata and M. Ioffe, J. Phys. A: Math. Gen. 34 (2001) 1129.

[3] L. D. Landau and E. M. Lifshitz, Quantum Mechanics, Pergamon, London, 1960, ch. V, par. 35.

[4] M. Znojil and M. Tater, J. Phys. A: Math. Gen. 34 (2001) 1793.

[5] E. Caliceti, S. Graffi and M. Maioli, Commun. Math. Phys. 75 (1980) 51;

V. Buslaev and V. Grechi, J. Phys. A: Math. Gen. 26 (1993) 5541;

C. M. Bender and K. A. Milton, Phys. Rev. D 55 (1997) R3255;

C. M. Bender and S. Boettcher, Phys. Rev. Lett. 24 (1998) 5243.

F. M. Fernández, R. Guardiola, J. Ros and M. Znojil, J. Phys. A: Math. Gen. 31 (1998) 10105.

[6] E. Delabaere and F. Pham, Phys. Lett. A 250 (1998) 25 and 29;

C. M. Bender, S. Boettcher and P. N. Meisinger, J. Math. Phys. 40 (1999) 2201;

A. A. Andrianov, M. V. Ioffe, F. Cannata and J. P. Dedonder, Int. J. Mod. Phys. A 14 (1999) 2675;

M. Znojil, F. Cannata, B. Bagchi and R. Roychoudhury, Phys. Lett. B 483 (2000) 284;

G. Lévai and M. Znojil, J. Phys. A: Math. Gen. 33 (2000) 7165 with further references. 
[7] S. Flügge, Practical quantum mechanics I, Springer, Berlin, 1971, p. 68;

S. Albeverio, F. Gestesy, R. Hoegh-Krohn and H. Holden, Solvable Models in Quantum Mechanics, Springer, Heidelberg, 1988.

[8] A. Gangopadhyaya, U. P. Sukhatme, Phys.Lett. A 224 (1996) 5.

[9] R. Dutt, A. Gangopadhyaya, C. Rasinariu and U. Sukhatme, New Solvable Singular Potentials, arXiv: hep-th/0011096.

[10] M. Znojil, Phys. Rev. A 61 (2000) 066101;

M. Znojil, Poeschl-Teller paradoxes, arXiv: math-ph/0102034

\section{Acknowledgement}

Work supported by the Czech GA AS, contracts No. A 1048004 and A 1048101.

\section{Figure captions}

Figure 1. The choice of coordinates for three particles

Figure 2. Graphical determination of the roots $\kappa=\sqrt{E}$ at $G_{1}=G_{2}=G_{3}=1$ 
Figure 1

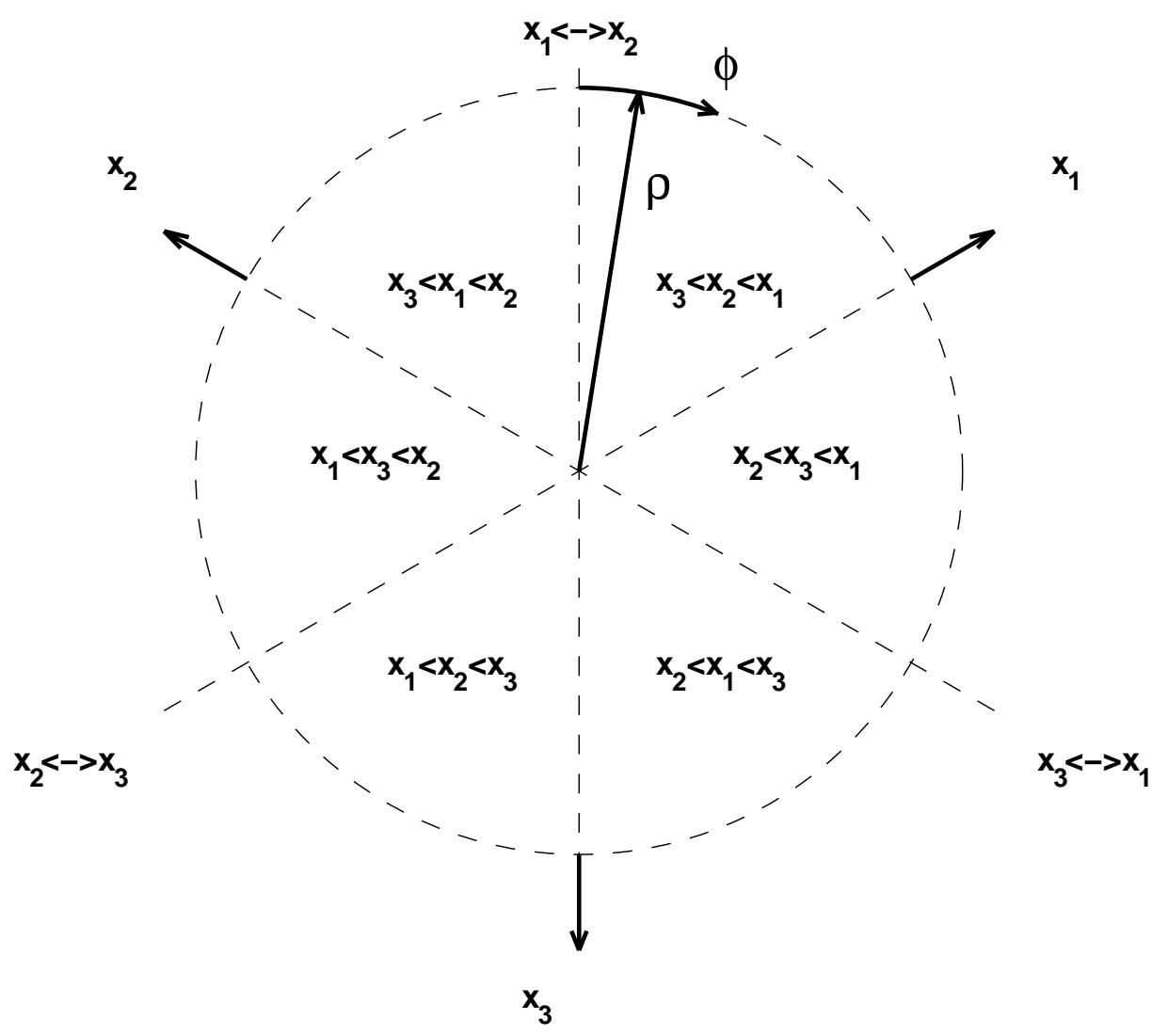


Figure 2

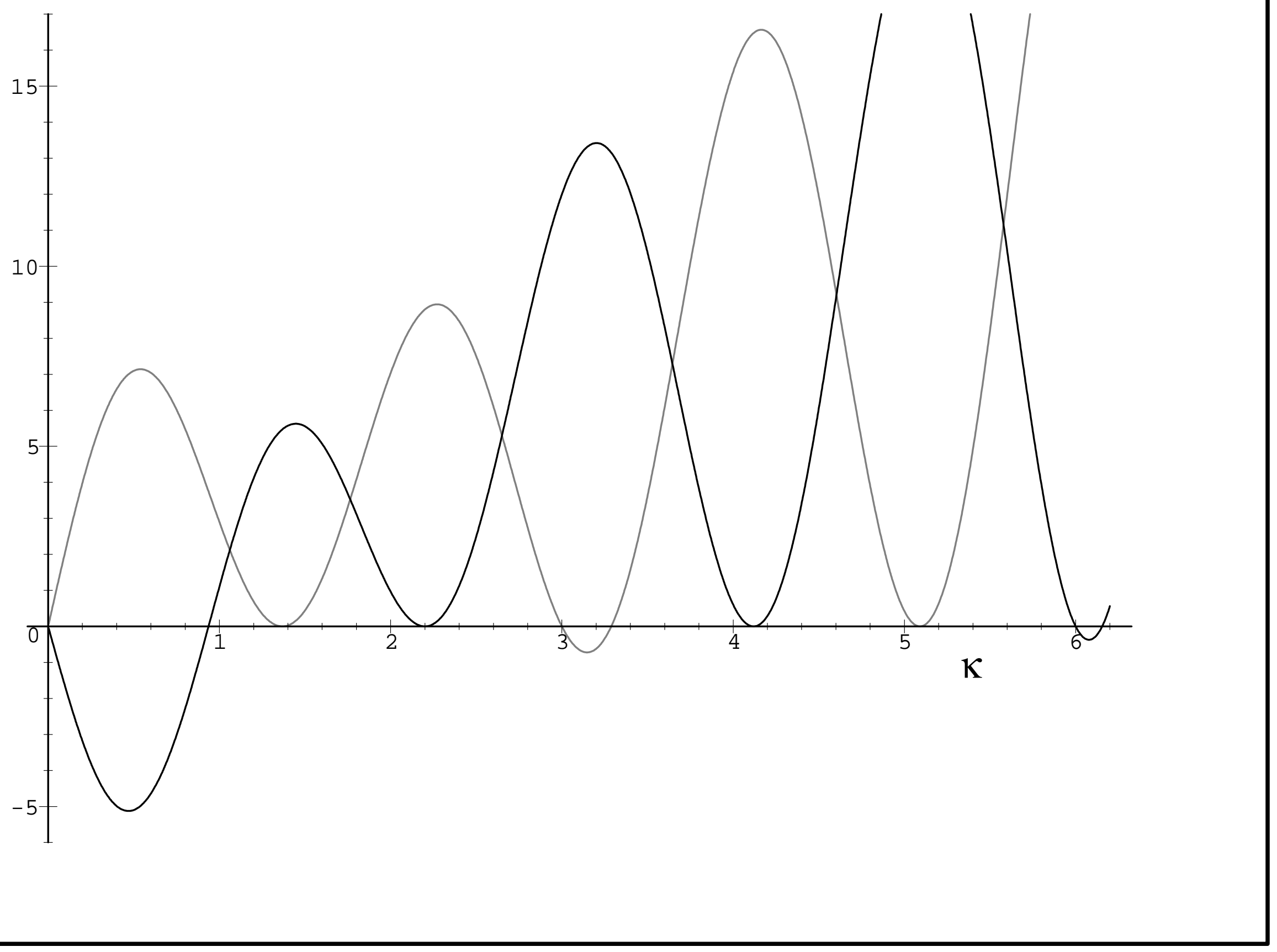




\title{
Exactly solvable three-body Calogero-type model with translucent two-body barriers
}

\author{
Miloslav Znojil and Miloš Tater \\ oddělení teoretické fyziky, \\ Ústav jaderné fyziky AV ČR, 25068 Řež, Czech Republic ${ }^{1}$
}

\begin{abstract}
A new exactly solvable alternative to the Calogero three-particle model is proposed. Sharing its confining long-range part, it contains the mere zero-range two-particle barriers. Their penetrability gives rise to a tunneling, tunable via their three independent strengths. Their variability can control the removal of the degeneracy of the energy levels in an innovative, non-perturbative manner.
\end{abstract}

PACS 21.45.+v 03.65.Ge 02.60.Lj

\footnotetext{
${ }^{1}$ e-mail: znojil@ujf.cas.cz and tater@ujf.cas.cz
} 


\section{Introduction}

In nuclear, atomic, molecular and statistical physics, the enormous popularity of the Calogero's solvable models [1] reflects a fairly realistic form of their separate twobody interactions. They combine a long-range quadratic attraction with a strong short-range repulsion. In its full generality the model binds an arbitary number $N$ of particles moving along a straight line and its exact solvability is a consequence of certain deep symmetries of its partial differential Schrödinger equation [2].

For the sake of brevity, let us pay attention just to the Calogero's first nontrivial three-body Hamiltonian

$$
\begin{aligned}
H^{(\text {Cal })}=-\frac{\hbar}{2 m}\left[\frac{\partial^{2}}{\partial x_{1}^{2}}+\frac{\partial^{2}}{\partial x_{2}{ }^{2}}+\frac{\partial^{2}}{\partial x_{3}^{2}}\right]+ \\
+\frac{1}{8} \omega^{2}\left[\left(x_{1}-x_{2}\right)^{2}+\left(x_{2}-x_{3}\right)^{2}+\left(x_{3}-x_{1}\right)^{2}\right]+ \\
+\left[\frac{g_{1}}{\left(x_{2}-x_{3}\right)^{2}}+\frac{g_{2}}{\left(x_{3}-x_{1}\right)^{2}}+\frac{g_{3}}{\left(x_{1}-x_{2}\right)^{2}}\right] .
\end{aligned}
$$

We may immediately see that from a purist's point of view, its two-body barriers are not penetrable [3] and, in this sense, they do not admit any mutual exchange of particles. For this reason we proposed recently a solvable modification of this model where a tunneling has been rendered possible at an expense of a loss of the Hermiticity of the Hamiltonian $H^{(\mathrm{Cal})}$ [4]. We employed a complexification based on the shift of the coordinates $x^{(\text {real })} \rightarrow x^{(\text {complex })}(t)=t-i \varepsilon\left(t^{2}\right)$. This replaced the Hermiticity of the Hamiltonian by its mere commutativity with the product of parity $\mathcal{P}$ and complex conjugation $\mathcal{T}[5]$. We have shown that the spectrum remained real in a way attributed to the $\mathcal{P} \mathcal{T}$ symmetry in the related literature [6].

In the latter innovative few-body implementation of the idea of the $\mathcal{P} \mathcal{T}$ symmetrization, the Calogero's strongly singular real barriers $1 / x^{2}$ with $x=x_{j}-x_{k}$ were all replaced by the complex and $\varepsilon$-dependent expressions

$$
\frac{1}{(x-i \varepsilon)^{2}}=\frac{1}{x^{2}+\varepsilon^{2}}+\frac{2 i \varepsilon x}{\left(x^{2}+\varepsilon^{2}\right)^{2}}+\mathcal{O}\left(\varepsilon^{2}\right) \text {. }
$$

In the present short note we intend to describe and analyze an alternative scheme 


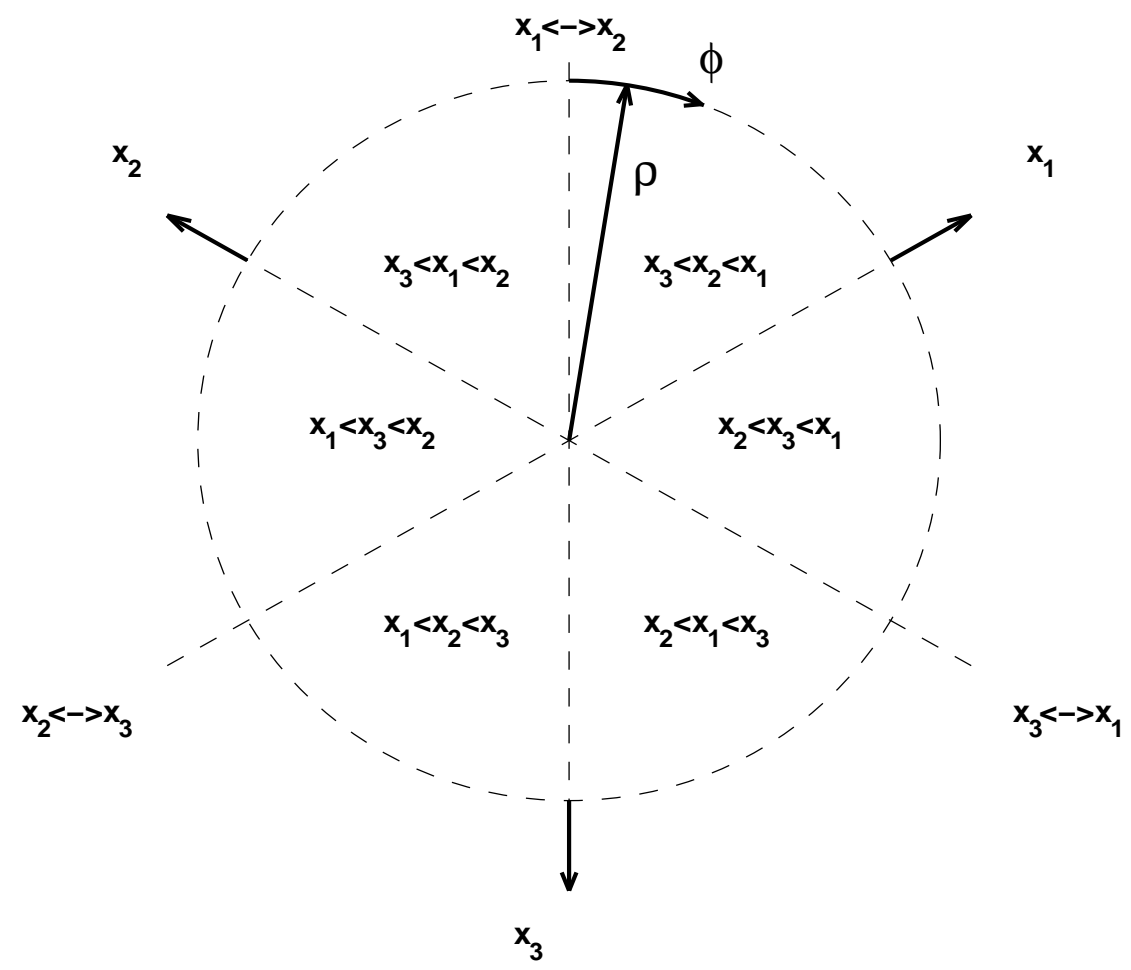

Figure 1: The choice of coordinates for three particles

which would admit a tunneling. In essence, we shall start from the same leading-order formula (2) but succeed in returning to a Hermitian Hamiltonian.

\section{The new model}

\subsection{Inspiration}

Our new proposal has been inspired by formula (2) and by its Hermitization

$$
\frac{1}{x^{2}} \rightarrow \frac{1}{x^{2}+\varepsilon^{2}}=\frac{1}{2 i \varepsilon}\left[\frac{1}{x-i \varepsilon}-\frac{1}{x+i \varepsilon}\right]=\frac{\pi}{\varepsilon} \tilde{\delta}_{\varepsilon}(x), \quad \varepsilon>0
$$


The limit $\varepsilon \rightarrow 0$ would then just reproduce the Calogero's model, one of the specific and most inspiring features of which lies in its separability in the three-body case. This is based on the re-parametrization of the coordinates $x_{1}-x_{2}=\sqrt{2} \rho \sin \phi$ with $\rho \in(0, \infty)$ and $\phi \in(0,2 \pi)$ etc (cf. Figure 1$)$. In the units $2 m=\hbar=1$ and for the equal and non-negative coupling constants $g_{1}=g_{2}=g_{3}=g \geq 0$ in eq. (1), such a change of variables reduces the Calogero's partial differential Schrödinger eqution to the mere ordinary Sturm - Liouvillean problem

$$
\left(-\frac{d^{2}}{d \phi^{2}}+\frac{9 g}{2 \sin ^{2} 3 \phi}\right) \psi(\phi)=\kappa^{2} \psi(\phi), \quad \psi(0)=\psi(2 \pi)=0 .
$$

It solution generates the well known (viz., Laguerre times Jacobi) polynomial wave functions as well as the related equidistant spectrum with a gap, $E_{n} \sim n+$ const, $n=0,2,3,4, \ldots$

\subsection{Main idea}

Our attention is attracted by the tilded expression $\tilde{\delta}_{\varepsilon}(x)$ in eq. (3). We shall try to re-read it as an approximate $\varepsilon \approx 0$ form of the well known Dirac delta function $\tilde{\delta}_{0}(x)=\delta(x)$. Thus, picking up, say, the first part of the two-body barrier in eq. (1) in its regularized form

$$
\frac{g_{1}}{\left(x_{2}-x_{3}\right)^{2}+\varepsilon^{2}}=f_{1} \tilde{\delta}_{\varepsilon}\left(x_{2}-x_{3}\right), \quad f_{1}=\frac{\pi g_{1}}{\varepsilon}, \quad \varepsilon>0,
$$

we may insert the appropriate definition $x_{2}-x_{3}=\sqrt{2} \rho \sin (\phi-4 \pi / 3)$ and get, at the very small $\varepsilon \rightarrow 0$,

$$
f_{1} \tilde{\delta}_{\varepsilon}\left(x_{2}-x_{3}\right) \approx \frac{G_{1}}{\rho^{2}}\left[\tilde{\delta}_{\varepsilon}\left(\phi-\frac{\pi}{3}\right)+\tilde{\delta}_{\varepsilon}\left(\phi-\frac{4 \pi}{3}\right)\right], \quad G_{1}=\frac{f_{1} \rho}{\sqrt{2}} .
$$

In place of (4) it gives

$$
\begin{aligned}
-\frac{d^{2}}{d \phi^{2}} \psi(\phi)+ & G_{1}\left[\tilde{\delta}_{\varepsilon}\left(\phi-\frac{\pi}{3}\right)+\tilde{\delta}_{\varepsilon}\left(\phi-\frac{4 \pi}{3}\right)\right] \psi(\phi)+ \\
+ & G_{2}\left[\tilde{\delta}_{\varepsilon}\left(\phi-\frac{2 \pi}{3}\right)+\tilde{\delta}_{\varepsilon}\left(\phi-\frac{5 \pi}{3}\right)\right] \psi(\phi)+ \\
& +G_{3}\left[\tilde{\delta}_{\varepsilon}(\phi-\pi)+\tilde{\delta}_{\varepsilon}(\phi)\right] \psi(\phi)=\kappa^{2} \psi(\phi),
\end{aligned}
$$

i.e., an approximative innovation of the Calogero's angular Schrödinger equation. 


\subsection{Interpretation}

Due to the purely intuitive form of the above "derivation" of eq. (5), one has to be very careful in all questions related to its possible physical as well as mathematical interpretation. At the same time, the use of the delta-function-shaped potentials is quite common in practice [7] as it makes many systems exactly solvable in the limit $\varepsilon \rightarrow 0$.

Let us start our further analysis of eq. (5) by picking up some three constants $G_{k}>0$. Then we re-define $f_{k}=f_{k}(\rho)=\sqrt{2} G_{k} / \rho$ and $g_{k}=\varepsilon f_{k}(\rho) / \pi$. In the generic case with $x_{i}-x_{j} \neq 0$, the limiting transition to $\varepsilon=0$ makes all the three centrifugallike forces vanish, $g_{k} \rightarrow 0$. Such an observation is compatible with the philosophy of using just the contact barriers in our angular Schrödinger equation.

In the second step we have to re-analyze the role of the overall singularity in the origin $\rho=0$. In principle, we might admit and consider many different singularities there [9]. Here we shall simplify our life by the most straightforward postulate that the point $\rho=0$ is just "regular" (i.e., "ignored" by our Hamiltonian), and that all our radial wave functions are simply vanishing in the origin. Under such an assumption, the final $\rho \neq 0$ form of our new Hamiltonian $H^{(n e w)}$ can be assigned a formal representation

$$
\begin{aligned}
H^{(\text {new })}=- & \frac{\hbar}{2 m}\left[\frac{\partial^{2}}{\partial x_{1}^{2}}+\frac{\partial^{2}}{\partial x_{2}^{2}}+\frac{\partial^{2}}{\partial x_{3}^{2}}\right]+ \\
+\frac{1}{8} \omega^{2} & {\left[\left(x_{1}-x_{2}\right)^{2}+\left(x_{2}-x_{3}\right)^{2}+\left(x_{3}-x_{1}\right)^{2}\right]+} \\
+ & \Omega_{1} \delta\left(x_{2}-x_{3}\right)+\Omega_{2} \delta\left(x_{3}-x_{1}\right)+\Omega_{3} \delta\left(x_{1}-x_{2}\right)
\end{aligned}
$$

where the strength of the two-body contact terms has the three-body character and weakens with the distance of the detached spectator particle,

$$
\Omega_{1}=\frac{\sqrt{3} G_{1}}{\left|x_{1}-x_{2}\right|}, \quad \Omega_{2}=\frac{\sqrt{3} G_{2}}{\left|x_{2}-x_{3}\right|}, \quad \Omega_{3}=\frac{\sqrt{3} G_{3}}{\left|x_{3}-x_{1}\right|} .
$$

We may summarize that our Hamiltonian $H^{(n e w)}$ represents a new three-particle model which is exactly solvable. 


\section{Solutions}

Our new angular Schrödinger equation

$$
\begin{aligned}
-\frac{d^{2}}{d \phi^{2}} \psi_{k}(\phi)+G_{1}\left[\delta\left(\phi-\frac{\pi}{3}\right)+\delta\left(\phi-\frac{4 \pi}{3}\right)\right] \psi_{k}(\phi)+ \\
+G_{2}\left[\delta\left(\phi-\frac{2 \pi}{3}\right)+\delta\left(\phi-\frac{5 \pi}{3}\right)\right] \psi_{k}(\phi)+ \\
+G_{3}[\delta(\phi-\pi)+\delta(\phi)] \psi(\phi)=\kappa_{k}^{2} \psi_{k}(\phi)
\end{aligned}
$$

describes the $\phi$-dependent part of the three-body wave function and determines its energies via the Calogero-type formula,

$$
E=E_{m, k}=\sqrt{\frac{3}{2}} \omega\left(2 m+1+\kappa_{k}\right), \quad m, k=0,1, \ldots
$$

Within the open subintervals of $\phi \in \mathcal{D}_{j}=([j-1] \pi / 3, j \pi / 3)$ we have a free motion

$$
\psi(\phi)=\psi_{j}(\phi)=A_{j} \sin \{\kappa[\phi-(j-1) \pi / 3]\}+B_{j} \cos \{\kappa[\phi-(j-1) \pi / 3]\}
$$

On the boundaries we have to match the separate local wave functions using the standard rules

$$
\psi_{j}\left(\frac{j \pi}{3}\right)=B_{j+1}, \quad \partial_{\phi} \psi_{j}\left(\frac{j \pi}{3}\right)=\kappa\left[A_{j+1}+\beta_{j}(\kappa) B_{j+1}\right], \quad j=1,2, \ldots, 6
$$

with $\beta_{j}(\kappa)=\beta_{j+3}(\kappa)=G_{j} / \kappa$ and $j=1,2$ or 3 . The first five applications of these rules define all the wave functions $\psi_{j}(\phi)$ in terms of the initial one, say, $\psi_{1}(\phi)$. The sixth step becomes a selfconsistent matching which guarantees that the global wave function remains single-valued. In terms of the two auxiliary matrices

$$
R=R(\kappa)=\left(\begin{array}{cc}
\cos (\kappa \pi / 3) & \sin (\kappa \pi / 3) \\
-\sin (\kappa \pi / 3) & \cos (\kappa \pi / 3)
\end{array}\right), \quad L=\left(\begin{array}{cc}
0 & 0 \\
1 & 0
\end{array}\right)
$$

our matching conditions factorize in the two independent two-dimensional forms

$$
(\mathcal{U} \pm I)\left(\begin{array}{c}
B_{1} \\
A_{1}
\end{array}\right)=0
$$


where $\mathcal{U}=\mathcal{U}(\kappa)=\left(I+\beta_{3} L\right) R\left(I+\beta_{2} L\right) R\left(I+\beta_{1} L\right) R$. The pertaining two alternative two-dimensional secular equations

$$
\operatorname{det}\left[\mathcal{U}\left(\kappa^{( \pm)}\right) \pm I\right]=0
$$

define all the roots $\kappa_{k}^{(\sigma)} \geq 0$ with the sign ambiguity $\sigma= \pm 1$ and the angular quantum number $k=0,1, \ldots$ in implicit manner.

\subsection{Toy model with the single barrier}

Let us fix $G_{1}=G_{2}=0$ and vary the strength $G=G_{3}$. With $\beta=G / \kappa^{(\sigma)}$ in the matrix

$$
\mathcal{U}^{(t o y)}(\kappa)=(I+\beta L) \cdot\left(\begin{array}{cc}
\cos \kappa \pi & \sin \kappa \pi \\
-\sin \kappa \pi & \cos \kappa \pi
\end{array}\right)
$$

our determinantal secular equation reads

$$
\cos \kappa \pi+\sigma+\frac{G \sin \kappa \pi}{2 \kappa}=0
$$

At $\sigma=-1$ and any integer shift of $\kappa^{(-)}=2 k+\delta$ with $k=0,1, \ldots$, this secular equation gives us two roots $\delta(a), \delta(b) \in[0,1)$. The smaller one is trivial, $\delta(a)=0$. The second one is uniquely specified by the implicit formula

$$
\tan \frac{\pi \delta(b)}{2}=\frac{G}{4 k+2 \delta(b)}
$$

Also for $\sigma=+1$ and $\kappa^{(+)}=2 k+1+\delta$ we get $\delta(a)=0$ and the similar relation

$$
\tan \frac{\pi \delta(b)}{2}=\frac{G}{4 k+2+2 \delta(b)}
$$

In the limit $G \rightarrow 0$ of the vanishing barrier we get the standard square-well solutions with the correct degeneracy $\delta(b) \rightarrow \delta(a)$ at every $k$.

The sign of the coupling depends on our choice, $G \in(-\infty, \infty)$. This provides an interesting counterpart to the Calogero model where the barrier cannot be too attractive [10]. In the limit $G \rightarrow \infty$ of the very strong repulsion, one returns to the Calogero-like case characterized by the impenetrability of the barriers. 


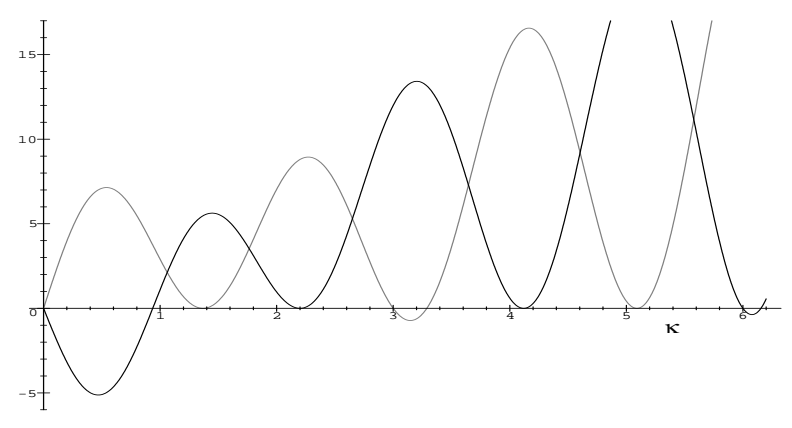

Figure 2: Graphical determination of the roots $\kappa=\sqrt{E}$ at $G_{1}=G_{2}=G_{3}=1$

\subsection{Three equal barriers}

When we choose $G_{1}=G_{2}=G_{3}=G$ and abbreviate $(I+\beta L) R=\mathcal{R}(\kappa)$ with $\beta=G / \kappa$, our secular equation (9) may be factorized into the two separate conditions at both the quasi-parities $\sigma= \pm 1$,

$$
\operatorname{det}[\mathcal{R}(\kappa)+\sigma I]=0, \quad \operatorname{det}\left[\mathcal{R}^{2}(\tilde{\kappa})-\sigma \mathcal{R}(\tilde{\kappa})+I\right]=0 .
$$

The first one parallels our preceding toy model and its solution is immediate,

$$
\kappa(a)=3 N, \quad \kappa(b)=3 N+3 \delta(b), \quad N=0,1, \ldots .
$$

The implicit definition of the pertaining shifts $\lambda(b) \in(0,1)$ has almost the same form as above,

$$
\tan \frac{\pi \delta(b)}{2}=\frac{G}{6 N+6 \delta(b)} .
$$

In the tilded case, the identity $\operatorname{det}\left(\mathcal{R}^{2}-\sigma \mathcal{R}+I\right)=(\sigma-\operatorname{tr} \mathcal{R})^{2}$ holds as long as $\operatorname{det} \mathcal{R}=1$. This reduces the second secular condition to the trigonometric equation

$$
2 \cos \frac{\tilde{\kappa} \pi}{3}+\frac{G}{\tilde{\kappa}} \sin \frac{\tilde{\kappa} \pi}{3}=\sigma .
$$

Its solutions must be sought numerically, giving $\tilde{\kappa}=1.367840720,2.199769250, \ldots$ at $G=1$ (cf. Figure 2). 


\section{Summary}

One of the most striking features of the model of Calogero is the fully impenetrable character of its two-body repulsive barriers. They divide the phase space in the six independent subdomains (cf. Figure 1). In a way, this absolute impenetrability of the barriers is in its effect responsible for the exact solvability of the Calogero's model.

This role of the Calogero's $g x^{-2}$ barriers is partially weakened when they become attractive, i.e., $0>g>-1 / 4$. An extension of the Calogero model to this transition region has been discovered and described by Gangopadhyaya and Sukhatme [8]. In their construction the contact terms also appeared as a formal means of preservation of the solvability. As a consequence, the spectrum only consisted of the two shifted sets of equally spaced energy levels. Such a type of the modified Calogero's spectrum re-appeared also in our recent non-Hermitian construction [4].

In the present letter we were able to get rid of the Calogero's power-law $\mathcal{O}\left(x^{-2}\right)$ barriers completely. Our alternative way of introduction of the contact terms enabled us to treat their couplings as independent parameters. Our key point is that these barriers are "thin", i.e., partially penetrable. This represents their main phenomenological appeal. At all their finite (and, in fact, both repulsive as well as attractive) couplings $G_{j}$, their free variability might prove appealing in many phenomenological considerations.

In contrast to the Calogero model characterized by the absolute absence of tunneling, all our particles are permitted to jump over one another. In certain applications to few body systems of quark type, this could improve our intuitive insight and build some analogies with the motion in more dimensions. After all, the use of the harmonic two-body forces with an additional, contact "local spike" might also extend the advantages of the exact solvability quite easily beyond the traditional domains in the theoretical nuclear physics. 


\section{References}

[1] F. Calogero, J. Math. Phys. 10 (1969) 2191 and 2197 and 12 (1971) 419.

[2] W. Rühl and A. Turbiner, Mod. Phys. Lett. A 10 (1995) 2213;

A. Turbiner, Mod. Phys. Lett. A 13 (1998) 1473;

A. Khare and C. Quesne, Phys. Lett. A 250 (1998) 33;

F. Cannata and M. Ioffe, J. Phys. A: Math. Gen. 34 (2001) 1129.

[3] L. D. Landau and E. M. Lifshitz, Quantum Mechanics, Pergamon, London, 1960, ch. V, par. 35.

[4] M. Znojil and M. Tater, J. Phys. A: Math. Gen. 34 (2001) 1793.

[5] E. Caliceti, S. Graffi and M. Maioli, Commun. Math. Phys. 75 (1980) 51;

V. Buslaev and V. Grechi, J. Phys. A: Math. Gen. 26 (1993) 5541;

C. M. Bender and K. A. Milton, Phys. Rev. D 55 (1997) R3255;

C. M. Bender and S. Boettcher, Phys. Rev. Lett. 24 (1998) 5243.

F. M. Fernández, R. Guardiola, J. Ros and M. Znojil, J. Phys. A: Math. Gen. 31 (1998) 10105.

[6] E. Delabaere and F. Pham, Phys. Lett. A 250 (1998) 25 and 29;

C. M. Bender, S. Boettcher and P. N. Meisinger, J. Math. Phys. 40 (1999) 2201;

A. A. Andrianov, M. V. Ioffe, F. Cannata and J. P. Dedonder, Int. J. Mod. Phys. A 14 (1999) 2675;

M. Znojil, F. Cannata, B. Bagchi and R. Roychoudhury, Phys. Lett. B 483 (2000) 284;

G. Lévai and M. Znojil, J. Phys. A: Math. Gen. 33 (2000) 7165 with further references. 
[7] S. Flügge, Practical quantum mechanics I, Springer, Berlin, 1971, p. 68;

S. Albeverio, F. Gestesy, R. Hoegh-Krohn and H. Holden, Solvable Models in Quantum Mechanics, Springer, Heidelberg, 1988.

[8] A. Gangopadhyaya, U. P. Sukhatme, Phys.Lett. A 224 (1996) 5.

[9] R. Dutt, A. Gangopadhyaya, C. Rasinariu and U. Sukhatme, New Solvable Singular Potentials, arXiv: hep-th/0011096.

[10] M. Znojil, Phys. Rev. A 61 (2000) 066101;

M. Znojil, Poeschl-Teller paradoxes, arXiv: math-ph/0102034

\section{Acknowledgement}

Work supported by the Czech GA AS, contracts No. A 1048004 and A 1048101.

\section{Figure captions}

Figure 1. The choice of coordinates for three particles

Figure 2. Graphical determination of the $\operatorname{roots} \kappa=\sqrt{E}$ at $G_{1}=G_{2}=G_{3}=1$ 